\title{
INHALT / SOMMAIRE
}

Herrn Prof. Dr. med. Erwin H. Ackerknecht zum 60. Geburtstag . . . 3

Publikationen von Prof. Dr. med. Erwin H. Ackerknecht . . . . . . . 5

Luigi Belloni, Appunti per una storia pre-Leeuwenhoekiana degli «animalcula». . . . . . . . . . . . . . . . . . . . . 13

Heinrich Buess, Die Anfänge der allgemeinen pathologischen Anatomie, insbesondere in der Pariser Schule . . . . . . . . . . . . . . . . . 23

Paul F. Cranefield, M.D., Ph.D., Freud and the "School of Helmholtz"

Robert Herrlinger, Bidloos «Anatomia» - Prototyp barocker Illustration?... . . . . . . . . . . . . . . . . . . . . . . . . .

E.Hintzsche, A.v. Hallers «Prospectus d'un dictionnaire universel de médecine». . . . . . . . . . . . . . . . . . . . . . . . . . . 48

R.HooykaAs, James Hutton und die Ewigkeit der Welt . . . . . . . . 55

Hans Jenzer, Die Gründung der Hebammenschulen in der Schweiz im 18. Jahrhundert . . . . . . . . . . . . . . . . . . . . . . .

Huldrych M. Koelbing, Alexander Spengler als Tuberkulose-Arzt und Klimatotherapeut . . . . . . . . . . . . . . . . . . . . . . . .

Fridolf Kudlien, Agrippa und Boerhaave: Zwei Positionen im Ringen um die certitudo medicinae . . . . . . . . . . . . . . . . . . . . 86

Erna Lesky, Kompensationslehre und denkökonomisches Prinzip . . . . 97

Nikolaus Mani, Paul Bert als Politiker, Pädagog und Begründer der Höhenphysiologie . . . . . . . . . . . . . . . . . . . . . .

G. De Morsier, Jean-Louis Prévost (1790-1850) et la découverte de l'ovule des mammifères. . . . . . . . . . . . . . . . . . . . . 117

EduARD-Rudolf Müllener, Zur methodischen therapeutisch-klinischen Forschung der «Ecole de Paris» (1800-1850) . . . . . . . . . . . . 122

George Rosen, Is Saul also among the Prophets? . . . . . . . . . . 132

K.E. Rотнаснuн, Zur Geschichte der physiologischen Reizmethodik im 17. und 18. Jahrhundert . . . . . . . . . . . . . . . . . . . . . 147

Gerhard Rudolph, Maine de Biran (1766-1824) . . . . . . . . . . . 161

Heinrich Schipperges, Wissenschaftsgeschichte und Kultursoziologie bei Ibn Chaldun . . . . . . . . . . . . . . . . . . . . . . . 170

Jean Starobinski, Note sur l'histoire des fluides imaginaires . . . . . . 176

Owser Temkin, Wunderlich, Schelling, and the History of Medicine . . . 188

JEAN THÉODORIDÈs, Les relations amicales de A. von Humboldt avec Guillaume Dupuytren . . . . . . . . . . . . . . . . . . 196

Hans H. Walser, Die wissenschaftlichen Anfänge von Adolf Meyer (1866-1950) und die Entstehung der «Zürcher psychiatrischen Schule» 5 3 8 5 6 17 2 47 . 70 176 